\title{
Aberrant epithelial differentiation by cigarette smoke dysregulates respiratory host defence
}

\author{
Gimano D. Amatngalim¹,3, Jasmijn A. Schrumpf ${ }^{1,3}$, Fernanda Dishchekenian¹, \\ Tinne C.J. Mertens ${ }^{1}$, Dennis K. Ninaber ${ }^{1}$, Abraham C. van der Linden ${ }^{1}$, \\ Charles Pilette ${ }^{2}$, Christian Taube ${ }^{1}$, Pieter S. Hiemstra (10) ${ }^{1}$ and \\ Anne M. van der Does ${ }^{1}$
}

Affiliations: ${ }^{1}$ Dept of Pulmonology, Leiden University Medical Center, Leiden, The Netherlands. ${ }^{2}$ Université Catholique de Louvain (UCL), Institut de Recherche Expérimentale \& Clinique (IREC), Pôle Pneumologie, ORL \& Dermatologie, Cliniques Universitaires St-Luc, Brussels, Belgium. ${ }^{3}$ Both authors contributed equally.

Correspondence: Anne M. van der Does, Dept of Pulmonology, Leiden University Medical Center, P.O. Box 9600, 2300 RC Leiden, The Netherlands. E-mail: a.van_der_doesalumc.nl

@ERSpublications

Loss of highly expressed host defence proteins as a result of cigarette smoke-induced airway epithelial remodelling http://ow.ly/Q6Jr30iR6Jg

Cite this article as: Amatngalim GD, Schrumpf JA, Dishchekenian F, et al. Aberrant epithelial differentiation by cigarette smoke dysregulates respiratory host defence. Eur Respir J 2018; 51: 1701009 [https://doi.org/10.1183/13993003.01009-2017].

ABSTRACT It is currently unknown how cigarette smoke-induced airway remodelling affects highly expressed respiratory epithelial defence proteins and thereby mucosal host defence.

Localisation of a selected set of highly expressed respiratory epithelial host defence proteins was assessed in well-differentiated primary bronchial epithelial cell (PBEC) cultures. Next, PBEC were cultured at the air-liquid interface, and during differentiation for 2-3 weeks exposed daily to whole cigarette smoke. Gene expression, protein levels and epithelial cell markers were subsequently assessed. In addition, functional activities and persistence of the cigarette smoke-induced effects upon cessation were determined.

Expression of the polymeric immunoglobulin receptor, secretory leukocyte protease inhibitor and long and short PLUNC (palate, lung and nasal epithelium clone protein) was restricted to luminal cells and exposure of differentiating PBECs to cigarette smoke resulted in a selective reduction of the expression of these luminal cell-restricted respiratory host defence proteins compared to controls. This reduced expression was a consequence of cigarette smoke-impaired end-stage differentiation of epithelial cells, and accompanied by a significant decreased transepithelial transport of IgA and bacterial killing.

These findings shed new light on the importance of airway epithelial cell differentiation in respiratory host defence and could provide an additional explanation for the increased susceptibility of smokers and patients with chronic obstructive pulmonary disease to respiratory infections. 


\section{Introduction}

Respiratory infections and microbial colonisation are a major health burden in smokers, and contribute to exacerbations and to the development and progression of chronic obstructive pulmonary disease (COPD) (reviewed by SETHI [1]). The mechanisms underlying this increased susceptibility of smokers with or without COPD are incompletely understood, but can be attributed in part to epithelial injury and remodelling resulting in a disrupted mucociliary clearance [2]. In addition to mucociliary clearance, the airway epithelium contributes to host defence with a wide variety of additional activities [3] that include secretion of antimicrobial peptides that act as endogenous antibiotics or modulate important antimicrobial immune responses via a variety of mechanisms [4]. Furthermore, the epithelium produces cytokines and chemokines that initiate an immune response to act against microbial invaders. Finally, transport of polymeric IgA and IgM to the lumen by the polymeric immunoglobulin receptor ( $\mathrm{pIgR}$ ) contributes to adaptive immunity in the lung by inhibiting adherence and facilitating clearance of pathogens, a process called immune exclusion [5]. Several of these respiratory host defence proteins (HDPs) in the airways are highly expressed during homeostasis by epithelial cells, suggesting their importance for airway epithelial barrier function. Highly expressed proteins and peptides include, but are not limited to antimicrobial peptides such as human $\beta$-defensin (hBD)-1 and lipocalin 2 (LCN2), the secretory leukocyte protease inhibitor (SLPI), pIgR and the epithelial sodium channel regulators short and long palate, lung and nasal epithelium clone protein (s/lPLUNC or BPIFA1/BPIFB1) [6-8]. Expression of other peptides involved in airway host defence such as ribonuclease (RNase)7, LL-37 and hBD-2 is low during homeostasis, but can be induced by inflammatory mediators, microbial products and upon injury of epithelial cells, and thus contributes to clearance of the pathogen and the resulting inflammatory process [4]. The pseudostratified airway epithelium is composed of several cell types, including goblet, club and ciliated cells that reach out toward the lumen of the airways, while basal cells do not reach this lumen in the intact epithelial layer [9]. Based on their distinct anatomical positioning, it is not surprising that these different cell types also produce different types of mediators. For example, expression of $\mathrm{pIgR}$ is restricted to the luminal cells of the pseudostratified airway epithelium and is therefore largely regulated by airway epithelial cell differentiation [10], similar to mucin production by goblet cells. In contrast, expression of the antimicrobial protein RNase 7 is restricted to basal cells [11].

Cigarette smoke is known to induce airway epithelial remodelling in smokers and patients with COPD, characterised by an increase in goblet cells and a reduction in presence of ciliated cells [2]. As a result, higher levels of mucus are produced by the epithelium, while mucus transport is impaired, thereby compromising mucociliary clearance activity of luminal airway epithelial cells. Currently it is unknown if the expression of proteins that are important for airway epithelial defence is polarised in the epithelium, and if so, how cigarette smoke-induced remodelling of the airway epithelium affects their expression. We hypothesised that cigarette smoke-induced alterations in epithelial cell differentiation result in a decreased expression of proteins that contribute to respiratory HDPs, which may render the host more susceptible to infection.

\section{Methods}

\section{Cell culture}

Primary bronchial epithelial cells (PBECs) were obtained from tumour-free resected lung tissue at the Leiden University Medical Center (Leiden, the Netherlands), as described in the online supplementary material, and cultured as described [11]. PBECs were cultured at the air-liquid interface (ALI) for 13-19 days (figure 1a). Apical washes were performed daily; medium was refreshed every other day.

\section{Fractionation of the airway epithelial cultures}

Luminal and basal cell-enriched fractions were obtained from 3-4 weeks differentiated ALI-PBEC cultures as described previously [11]. The luminal cell fraction was spun down and either lysed in RNA lysis buffer or fixed with $1 \%$ paraformaldehyde (Millipore, Amsterdam, the Netherlands) in PBS for $10 \mathrm{~min}$ on ice and washed afterwards in ice-cold PBS. The remaining basal epithelial cell fractions on the transwell inserts were either lysed in RNA lysis buffer (Promega, Leiden) or fixed with $1 \%$ paraformaldehyde (Millipore) in PBS for $10 \mathrm{~min}$ on ice and washed afterwards with ice-cold PBS. Next, cells were stained as described in the online supplementary material with antibodies described in online supplementary table S2.

\section{Chronic cigarette smoke exposure}

When confluent, PBECs were air-exposed (day 0) by removal of medium from the apical side of the transwell insert and $4 \mathrm{~h}$ later exposed to freshly generated whole cigarette smoke using $3 \mathrm{R} 4 \mathrm{~F}$ reference cigarettes (University of Kentucky, Lexington, KY, USA). Cigarette smoke exposure was repeated daily as described in [11], in the figure legends of figure 1 and online supplementary figure S1 and illustrated in 
a)

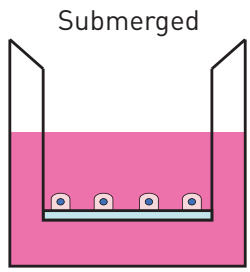

Flow

b)

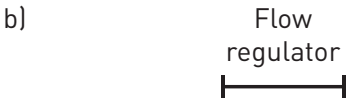

Air-exposed

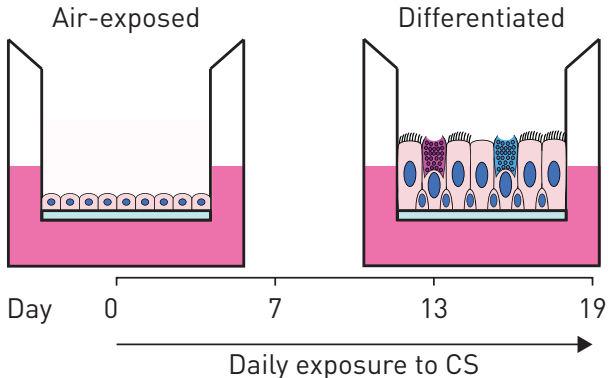

Daily exposure to CS

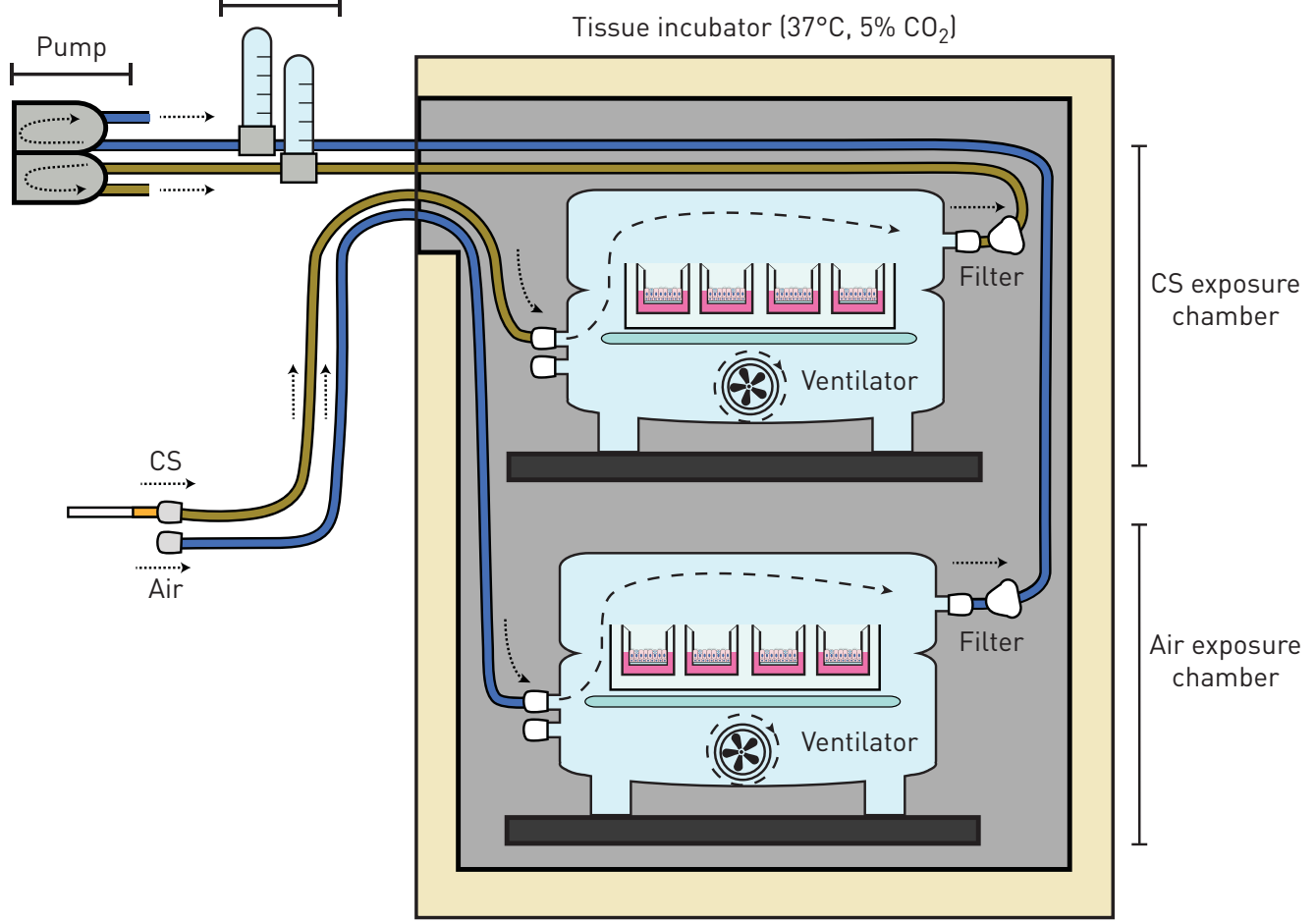

FIGURE 1 Cell culture set-up and cigarette smoke (CS) exposure of primary bronchial epithelial cells (PBECs) differentiated at the air-liquid interface. a) PBECs were seeded on coated transwells and cultured in submerged conditions until confluent. At day 0 , cultures were air-exposed and cultured for an additional 1319 days to allow mucociliary differentiation. b) Each day, starting at day 0, cultures were exposed to CS by placing them in an exposure chamber that was infused with either CS or with air for 4-5 min. Next, residual smoke in the chamber was removed for a period of $10 \mathrm{~min}$ by infusing the chambers with air derived from the incubator. $\sim 4 \mathrm{~h}$ before each CS exposure the apical surface of the cultures was washed to remove mucus. Basal medium was changed every other day. $\mathrm{CO}_{2}$ : carbon dioxide.

figure $1 \mathrm{~b}$ and online supplementary figure S1. Briefly, cells were exposed in modified hypoxic chambers for 4-5 min to either cigarette smoke from one cigarette or to room air, after which smoke was removed by ventilation with air over $10 \mathrm{~min}$ and cells were subsequently placed back in the incubator overnight. 18-20 h later, ALI-PBEC were washed apically with PBS and $4 \mathrm{~h}$ thereafter exposed to cigarette smoke. This cycle was repeated every day until day 13-19. Cells were harvested for analysis $18-20 \mathrm{~h}$ after the last cigarette smoke exposure.

RNA isolation, complementary DNA synthesis and quantitative PCR

Methods are described in the online supplementary material with primers described in online supplementary table S1.

\section{Confocal microscopy}

Cells were fixed on transwell inserts in 1\% paraformaldehyde (Millipore) in PBS for $10 \mathrm{~min}$ on ice and washed afterwards with ice-cold PBS. Next, cells were stained as described in the online supplementary material with antibodies described in online supplementary table S2. 
Transcytosis experiment

Methods are described in the online supplementary material.

Antibacterial activity assay

Methods are described in the online supplementary material

ELISA and transepithelial electrical resistance

Methods are described in the online supplementary material.

\section{Inhibition of differentiation by DAPT}

At day 0, PBECs were air-exposed by removal of the medium in the insert and culture medium of ALI-PBEC was refreshed with medium supplemented with either $5 \mu \mathrm{M}$ DAPT (Notch signalling inhibitor; Sigma Aldrich, Zwijndrecht, the Netherlands) or solvent control. Every other day, basal medium was changed in a similar fashion up to day 13 , when the cells were harvested.

\section{Statistics}

Statistical analysis was conducted using GraphPad Prism 7 (GraphPad Software, La Jolla, CA, USA). Data are shown as mean \pm SEM and significance was tested using a paired t-test or two-way ANOVA with a Bonferroni-corrected post hoc test. Differences were considered significant at $\mathrm{p}<0.05$.

\section{Results \\ Respiratory host defence proteins display a polarised distribution in airway epithelial cell cultures \\ In this study we have focused on a set of proteins and peptides that are important for respiratory host defence. These HDPs were selected based on their constitutive and/or high expression by airway epithelial cells during homeostasis, i.e. SLPI, s/IPLUNC, pIgR, hBD-1 and LCN2. First, we investigated whether expression of these proteins was polarised in the airway epithelial cultures. To this end, we prepared luminal and basal epithelial cell-enriched fractions of well-differentiated PBECs, cultured at the ALI (figure 2a). We confirmed the successful enrichment of fractions for luminal and basal cells by determining the gene expression of the typical basal cell markers TP63 and KRT5 and luminal epithelial cell markers FOXJ1 (ciliated cells), SCGB1A1 (club cells), MUC5AC and MUC5B (both goblet cells) (figure 2a), and by immunofluorescence staining for p63 (basal cells), CC16 (club cells) and acetylated $\alpha$-tubulin (ciliated cells) (online supplementary figure S2). Further analysis of these fractions showed that the luminal cell-enriched fraction expressed significantly higher levels of BPIFA1 (sPLUNC), BPIFB1 (IPLUNC) and SLPI (figure 2a). In contrast, LCN2 and DEFB1 expression did not differ between the luminal and basal cell-enriched fraction (figure 2a). The luminal cell-specific expression of SLPI and sPLUNC was further confirmed using confocal imaging, in which the staining of both proteins did not colocalise with $\mathrm{p} 63^{+}$basal cells, but was highly present at the apical side of the PBEC culture and in the luminal cell-enriched fraction (figure $2 \mathrm{~b}$ and online supplementary figure S2).}

\section{Chronic cigarette smoke exposure of airway epithelial cell cultures reduces expression of respiratory HDPs}

Next, we investigated if cigarette smoke exposure affected expression of this set of respiratory HDPs. To this end, ALI-PBEC cultures were exposed on a daily basis during 2-3 weeks of differentiation to whole cigarette smoke (figure 1 and online supplementary figure S1). Gene expression analysis showed that DEFB1 (hBD-1) mRNA levels decreased during differentiation, but were not affected by cigarette smoke exposure (figure 3a). Conversely, expression of SLPI, BPIFA1 (sPLUNC), BPIFB1 (IPLUNC) and PIGR strongly increased during differentiation, and this increase was significantly prevented by cigarette smoke (figure 3a). In contrast, gene expression of LCN2 (lipocalin 2) was increased by cigarette smoke exposure during differentiation (figure 3a). These findings were further confirmed by assessment of hBD-1 and SLPI protein levels in the apical wash and in basal medium from the ALI-PBEC cultures (figure $3 b$ ). Indeed, hBD-1 levels reduced over the time of differentiation in the apical wash, but were not significantly affected by chronic cigarette smoke exposure, whereas SLPI levels were significantly lower in chronic cigarette smoke-exposed cell cultures (figure 3b). Next, we performed immunofluorescence staining of the airway epithelial cell cultures and found strongly reduced presence of SLPI-, sPLUNC- and pIgR-positive cells in chronic cigarette smoke-exposed epithelium compared to air controls (figure $3 \mathrm{c}$ ). These results confirmed selective impairment of specific respiratory HDPs by chronic cigarette smoke exposure during airway epithelial differentiation. 
a)
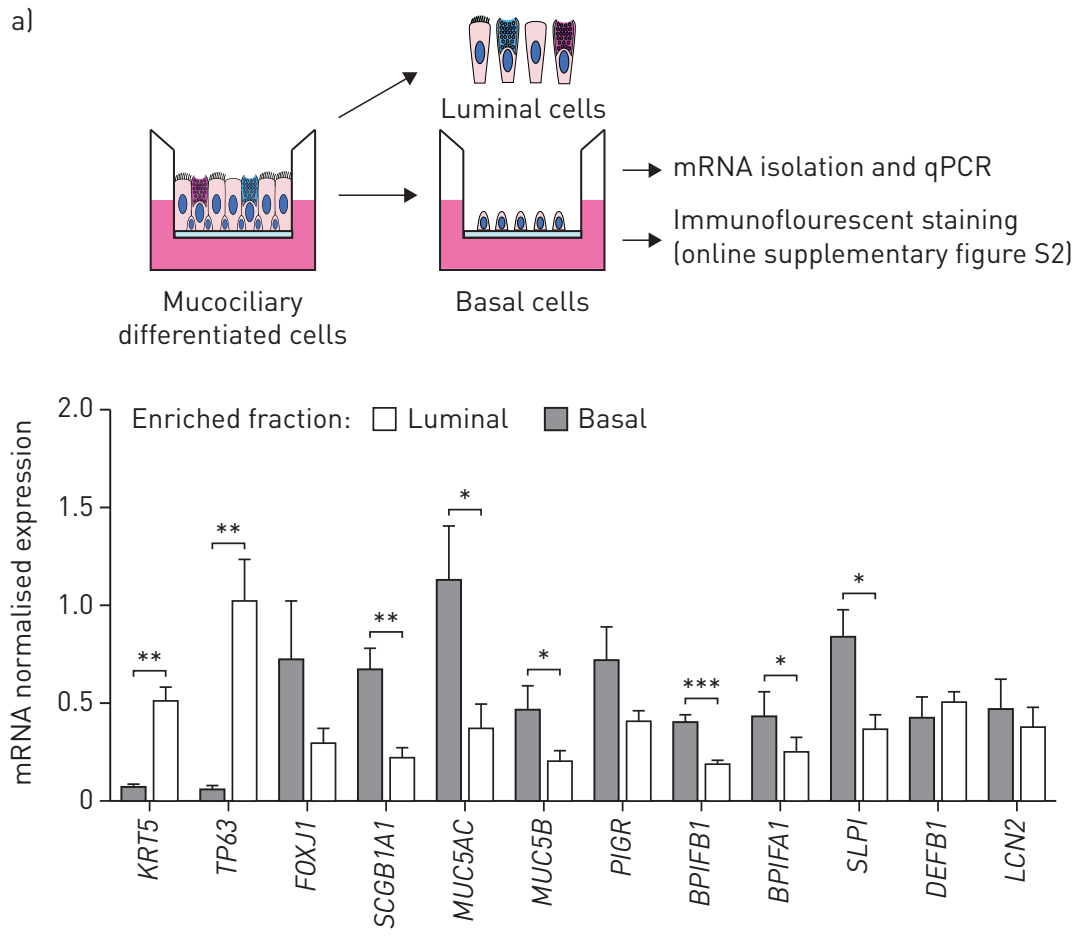

b)
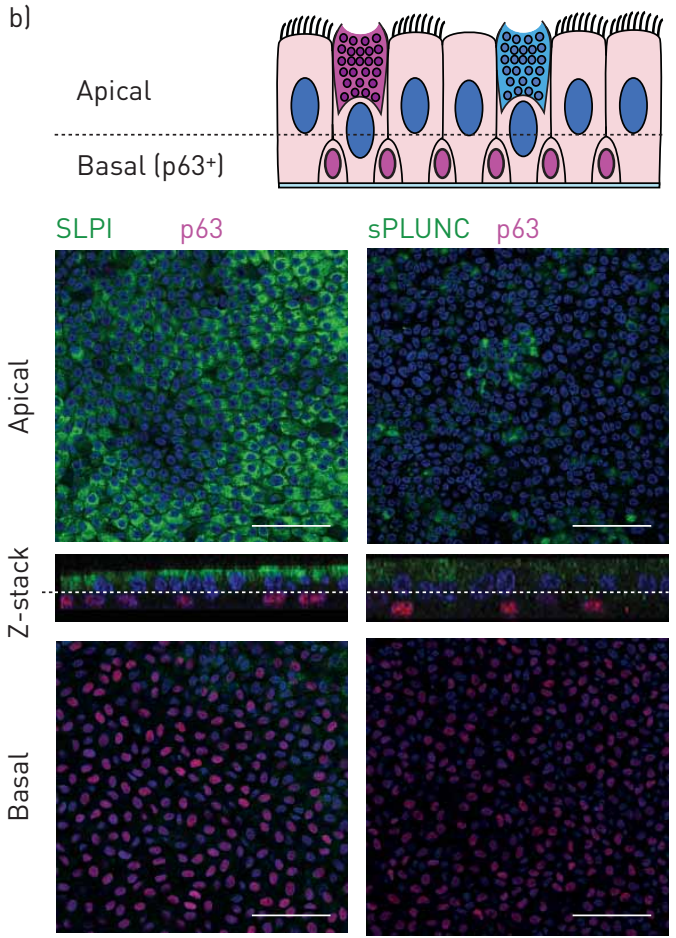

SPLUNC p63

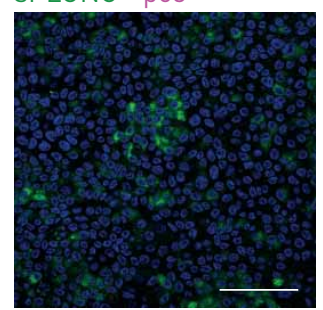

FIGURE 2 Respiratory host defence proteins display a polarised distribution in air-liquid interface (ALI) cultures of primary bronchial epithelial cells (PBECs). a) PBECs were seeded on coated transwells and cultured in submerged conditions until confluent. At day 0 , cultures were air-exposed and cultured at the ALI. After 3-4 weeks of differentiation luminal and basal cell-enriched fractions were separated followed by RNA isolation, complementary DNA synthesis and quantitative (q)PCR analysis. Data are shown as target gene expression normalised for the geometric mean expression of the reference genes ATP synthase, hydrogen transporting, mitochondrial F1 complex, $\beta$-polypeptide (ATP5B), $\beta 2$-microglobulin $(B 2 M)$ and ribosomal protein L13a (RPL13A); $\mathrm{n}=5-7$ different donors. Statistical significance was tested using a paired t-test. *: $p<0.05,{ }^{* *}: p<0.01,{ }^{* *}: p<0.001$. b) Confocal images to visualise polarised distribution of secretory leukocyte protease inhibitor (SLPI) and short palate, lung and nasal epithelium clone protein (SPLUNC) in differentiated ALI-PBEC culture cells. After 3 weeks of differentiation, cells were fixed in 1\% paraformaldehyde and stained using immunofluorescence with primary antibodies against p63 (basal cell marker, red) in combination with primary antibodies against SLPI and/or sPLUNC (both green) and 4',6-diamidino-2-phenylindole (DAPI) for nuclear staining (blue). Z-stacks and images of the apical and basal side of stained cells were made by confocal imaging. Images shown are representative for results obtained with cells from four different donors. Scale bars $=50 \mu \mathrm{m}$.

\section{Chronic cigarette smoke exposure reduces host defence of the airway epithelial cell cultures by decreasing apical release of secretory IgA and bacterial killing of Moraxella catarrhalis and Klebsiella pneumoniae}

Next, we assessed whether the strong reduction in SLPI, BPIFA1 (sPLUNC), BPIFB1 (IPLUNC) and PIGR expression levels in the cigarette smoke-exposed airway epithelial cultures had functional consequences for host defence. We selected pIgR-mediated transfer of dimeric (d)IgA across the epithelium as a proof-of-principle for the consequences on immunomodulatory host defence functions and found this to be significantly reduced in chronic cigarette smoke-exposed cultures (figure 4a). Furthermore, we analysed bacterial killing by chronic cigarette smoke-exposed cell cultures of the Gram-negative bacteria Moraxella catarrhalis and Klebsiella pneumoniae, pathogens that are found to be increased in the lungs of patients with stable or acute exacerbations of COPD [12]. We observed significantly higher bacterial counts (indicating lower antibacterial activity) in chronic cigarette smoke-exposed PBEC cultures when compared to air-exposed cultures for both pathogens (figure $4 \mathrm{~b}$ ). These data indicate that various host defence mechanisms are functionally impaired in cigarette smoke-exposed epithelial cell cultures, which corresponds with impaired expression of respiratory defence proteins.

\section{Cigarette smoke affects end-stage differentiation of airway epithelial cells}

Next, we assessed whether chronic cigarette smoke exposure affected differentiation of ALI-PBECs by measuring gene expression of epithelial cell-specific markers. Gene expression of the basal cell markers cytokeratin-5 (KRT5) and TP63 and of cytokeratin-8 (KRT8), which is expressed by intermediate/ committed progenitor epithelial cells [13], was not affected by cigarette smoke (figure 5a). In contrast, expression of the specialised luminal epithelial cell-specific genes FOXJ1 (ciliated cells), SCGB1A1 (club cells) and MUC5B (goblet cells) increased during differentiation, and this increase was significantly prevented by cigarette smoke (figure 5a). Confocal imaging confirmed the aberrant epithelial 

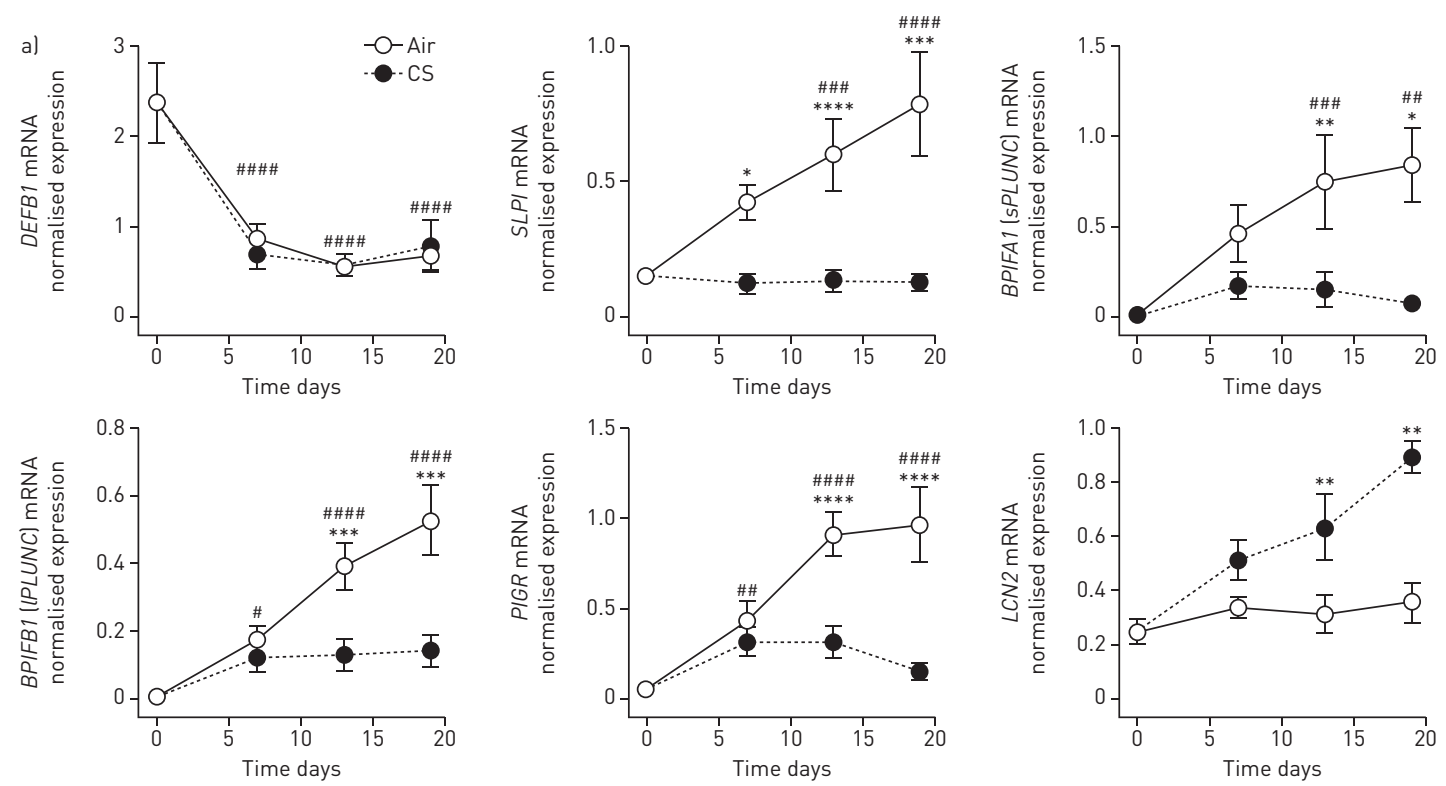

b)
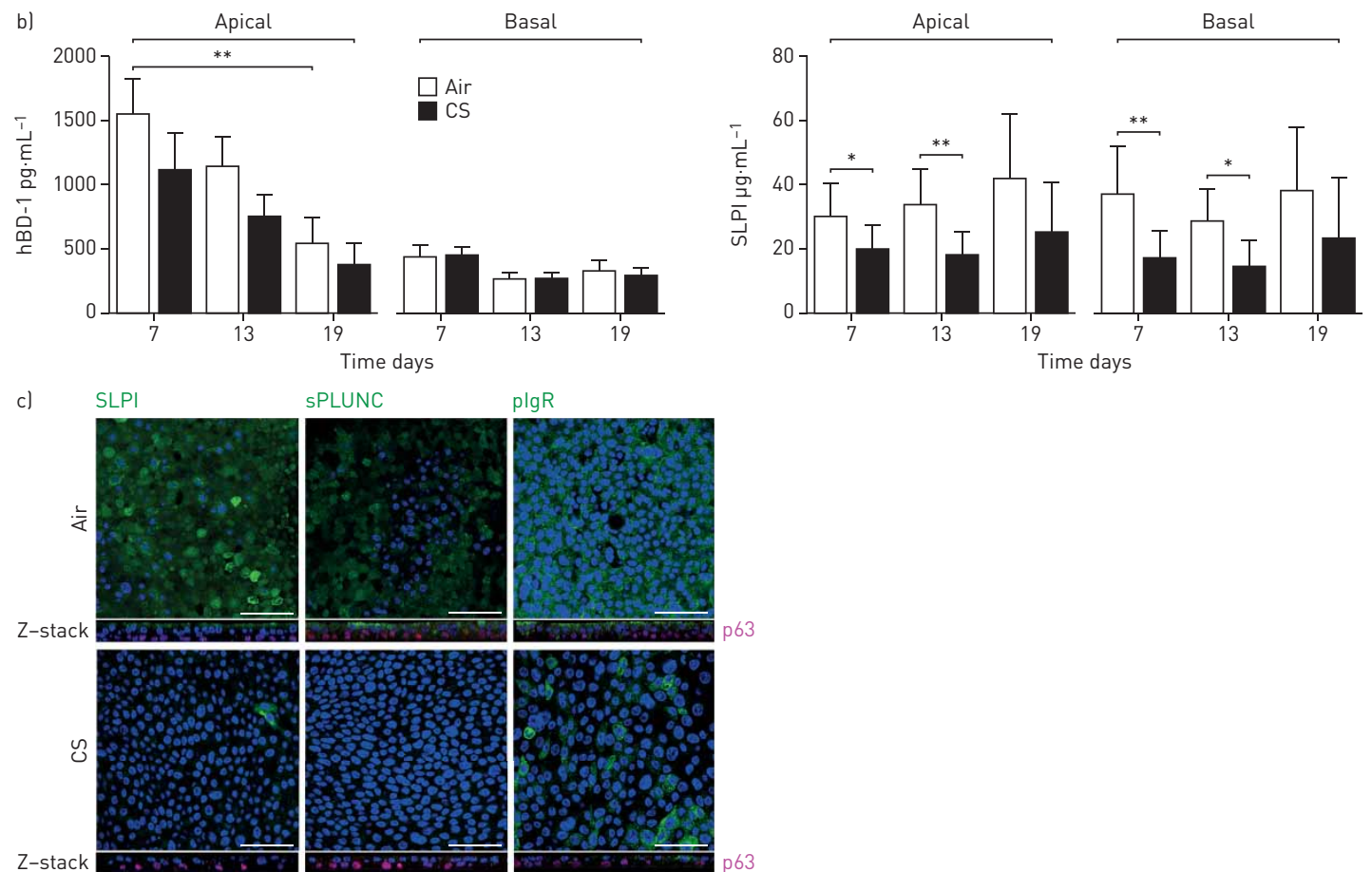

plgR

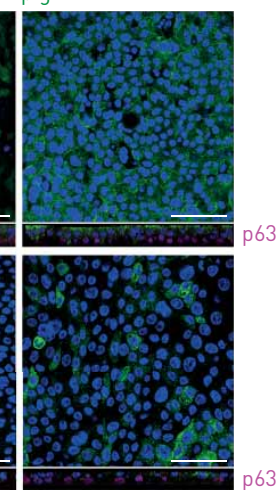

FIGURE 3 Chronic cigarette smoke exposure of air-liquid interface (ALI) cultures of primary bronchial epithelial cells (PBECs) lowers the expression of luminal cell-restricted host defence proteins. a) ALI-PBECs were exposed daily to whole cigarette smoke (CS) or air as a control

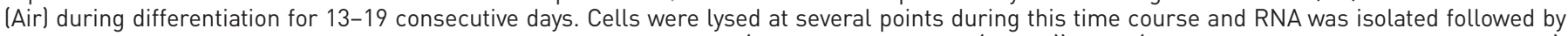
complementary DNA synthesis to assess gene expression of DEFB1 (human $\beta$-defensin-1 (hBD-1)), SLPI (secretory leukocyte protease inhibitor), BPIFA1 (short palate, lung and nasal epithelium clone protein (sPLUNC)), BPIFB1 (long palate, lung and nasal epithelium clone protein (IPLUNC)), $P I G R$ (polymeric immunoglobulin receptor) and LCN2 (lipocalin 2). Data are presented as target gene expression normalised for the geometric mean expression of the reference genes ATP synthase, hydrogen-transporting, mitochondrial F1 complex, $\beta$ polypeptide (ATP5B), $\beta 2$-microglobulin $(B 2 M)$ and ribosomal protein L13a (RPL13A). Days 0, 7 and $13 \mathrm{n}=8$ different donors; day $19 \mathrm{n}=4$ different donors. Statistical differences were evaluated using a two-way ANOVA and Bonferroni post hoc test. ${ }^{*}:$ p $<0.05, * *: p<0.01,{ }^{* * *}:$ p $<0.001,{ }^{* * * *}:$ p<0.0001 between air and CS. ${ }^{\#}$ : $p<0.05$, \#\#: $\mathrm{p}<0.01, \# \#$ : $\mathrm{p}<0.001, \# \# \#$ : $\mathrm{p}<0.0001$ between air at days 7,13 and 19 and unexposed cultures at day 0. b) ELISA for hBD-1 and SLPI was performed on the apical wash and basal medium of these cultures. Days 7 and 13 , $n=8$ different donors and day $19 \mathrm{n}=4$ different donors. Statistical differences on day 7 and day 13 (not day 19) was tested using a paired two-way ANOVA to compare air and CS. *: p<0.05, **: p<0.01. c) ALI-PBECs were differentiated for 2-3 weeks in which they were daily exposed to CS or air as a control. Subsequently, the cells were fixed in $1 \%$ paraformaldehyde and stained using primary antibodies against SLPI, sPLUNC and plgR lall green stainingl in combination with 4',6-diamidino-2-phenylindole (DAPI) to stain the nuclei (blue staining). Images shown are representative for results obtained with cell cultures from four different donors. Scale bars $=50 \mu \mathrm{m}$. 

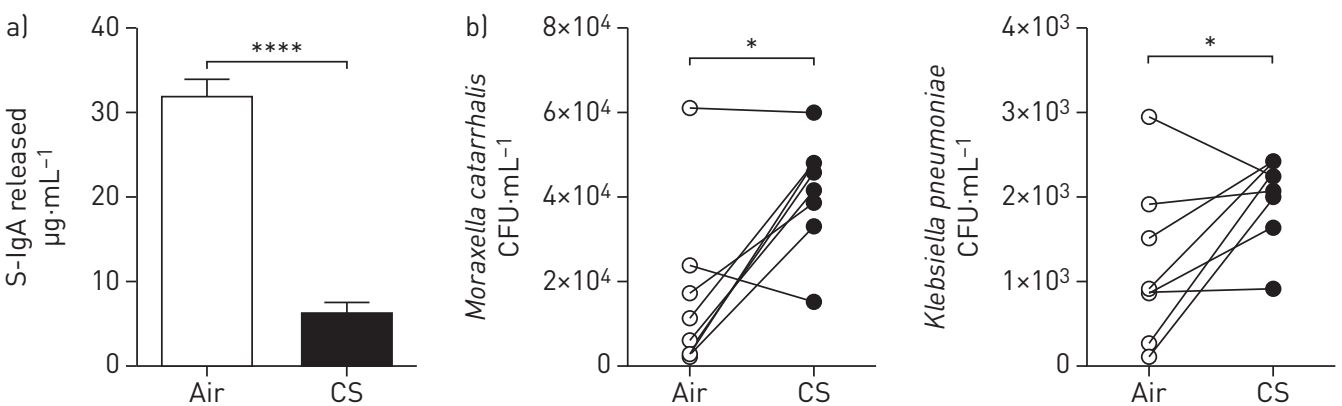

FIGURE 4 Chronic cigarette smoke exposure of air-liquid interface (ALI) cultures of primary bronchial epithelial cells (PBECs) impairs host defence activities. a) ALI-PBECs were exposed daily to whole cigarette smoke (CS) or air as a control (Air) during differentiation for 13 consecutive days. After 13 days of chronic CS exposure, dimeric (d) IgA transcytosis capacity of the epithelial cultures was assessed by determining secretory (S)-IgA levels in apical washes by ELISA (no S-IgA could be detected in the basal medium, as a control of the assay that does only recognise $S-\lg A$ and not $d-\lg A)$. $n=10$ different donors. b) After 13 days of chronic CS exposure, ALI-PBECs were cultured for $48 \mathrm{~h}$ in antibiotics-free cell culture medium after which they were exposed for $2 \mathrm{~h}$ to Moraxella catarrhalis or Klebsiella pneumoniae at the apical surface of the ALI-PBEC. The surviving bacteria are depicted as CFU $\mathrm{mL}^{-1} \cdot \mathrm{n}=8$ different donors. Significance was determined using a paired t-test. ${ }^{*}: p<0.05,{ }^{* * * *}: p<0.0001$.

differentiation in cigarette smoke-exposed cultures as cells positive for cilia marker acetylated $\alpha$-tubulin, the club cell marker CC16, and the goblet cell marker MUC5AC were reduced in chronic cigarette smoke-exposed cultures, while cytokeratin-8 $(\mathrm{CK}-8)^{+}$and $\mathrm{p}^{+} 3^{+}$cells remained unchanged between air- and cigarette smoke-exposed cultures (figure 5b).

\section{Reversibility of cigarette smoke-induced effects on HDP expression}

To assess the persistence of the cigarette smoke-induced reduction in SLPI, BPIFA1 (sPLUNC), BPIFB1 (IPLUNC) and PIGR expression levels and its effect on cellular composition, we allowed the cultures to recover from 13 days of cigarette smoke exposure by culturing the cells for an additional 6 days without cigarette smoke exposure. Chronic cigarette smoke-exposed cultures were able to (partly) recover from the lack of end-stage differentiation, since all specialised luminal cell markers, except for SCGB1A1 (club cells), significantly increased in expression (figure 6a). Furthermore, in addition, SLPI, BPIFA1 (sPLUNC), BPIFB1 (IPLUNC) and PIGR showed enhanced expression compared to day 13 (figure 6a). Additionally, KRT5 (basal cell marker), and DEFB1 and LCN2 increased upon cigarette smoke cessation, whereas TP63 and KRT8 were unaffected (online supplementary figure S3A). This indicates that the inhibitory effects of cigarette smoke exposure on epithelial differentiation and expression of specific respiratory defence proteins are at least in part reversible. Furthermore, in an attempt to better mimic the in vivo situation and establish whether the effects observed after chronic cigarette smoke exposure can be obtained when exposing an already partly differentiated epithelium to chronic cigarette smoke, we performed a separate experiment. In this experiment, we first allowed the cultures to differentiate for 1 week, after which we started chronic cigarette smoke exposure for an additional 12 days. Here we found similar effects of cigarette smoke exposure on ALI-PBEC cultures regarding cell-type specific markers and HDP expression compared to cigarette smoke exposure starting from day 0 (figure $6 \mathrm{~b}$ and online supplementary figure S3B).

\section{Notch signalling inhibition impairs HDP expression during differentiation}

Our results so far showed an impaired end-stage differentiation into specialised luminal epithelial cells in cigarette smoke-exposed cultures resulting in reduced levels of SLPI, sPLUNC, IPLUNC and pIgR. Previous studies have shown that Notch signalling is involved in airway epithelial differentiation and that the airway epithelium of smokers displays reduced Notch signalling [14]. Therefore, we next examined if Notch signalling was impaired in cigarette smoke-exposed cultures, and if Notch signalling inhibition modulates HDP expression during differentiation. First, we assessed gene expression of components of the Notch signalling cascade and found that chronic cigarette smoke exposure did not influence gene expression of Notch ligands, receptors or transcriptional co-activators in these cultures (figure $7 \mathrm{a}$ ). However, the Notch signalling target genes, HEY1 and HEY2, were significantly reduced by chronic cigarette smoke exposure, while HES1 was not (figure 7b), indicating that chronic cigarette smoke exposure selectively affects target genes of the Notch signalling pathway.

To further investigate the importance of Notch signalling in the expression of host defence proteins, we examined the effect of the $\gamma$-secretase inhibitor DAPT, which acts as an inhibitor of Notch 

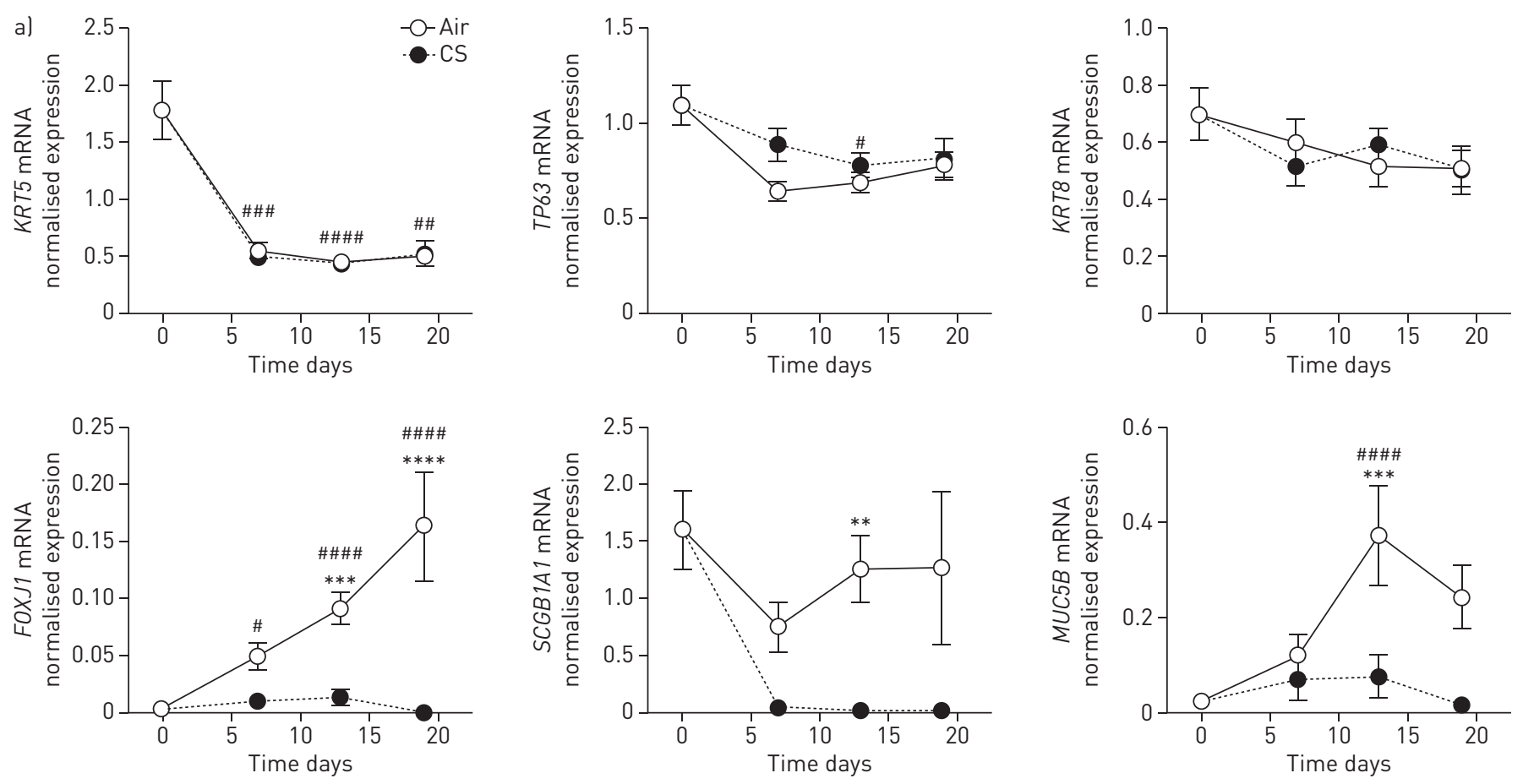

b)

p63

CK-8
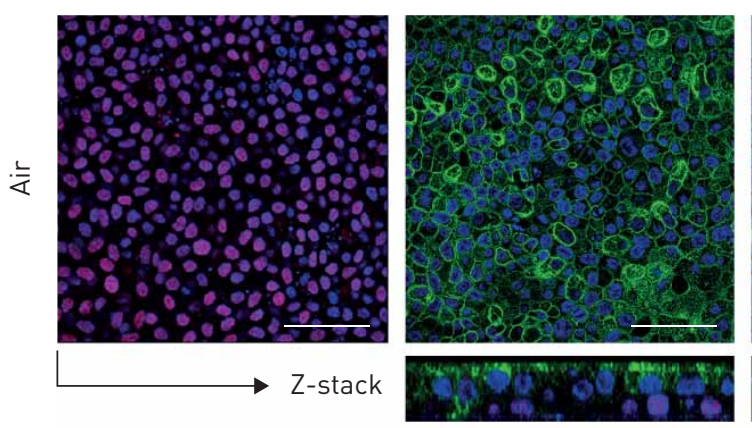

Acetylated $\alpha$-tubulin

CC16

MUC5AC
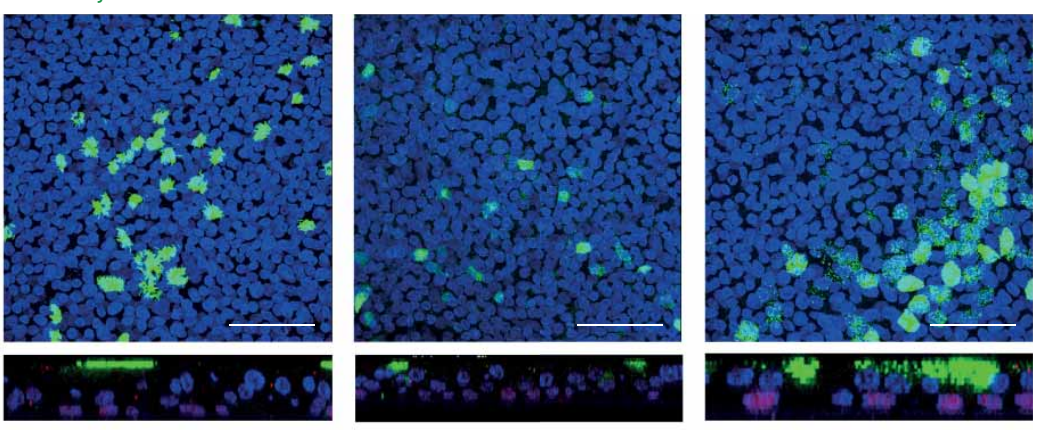

凹
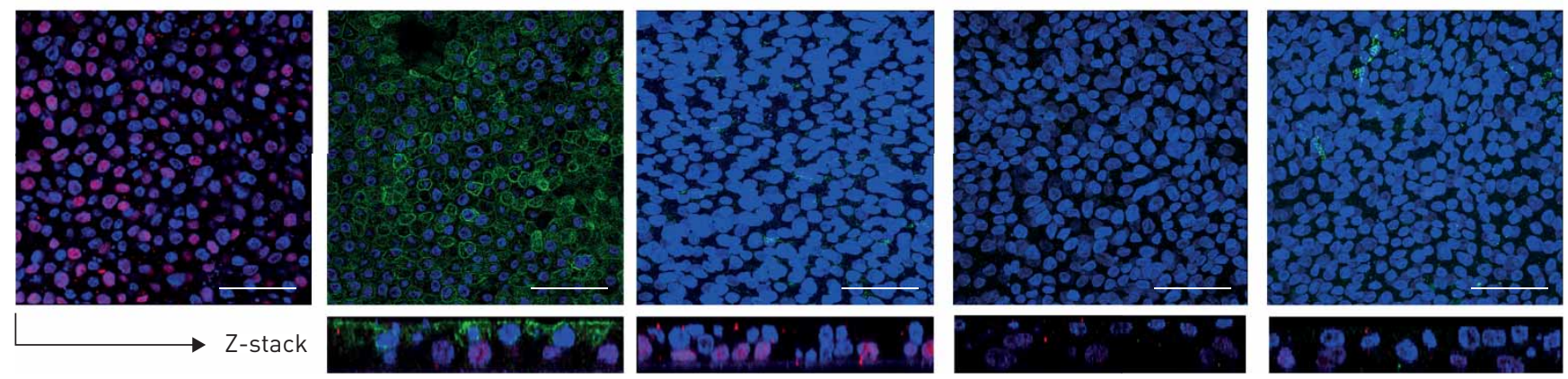

FIGURE 5 Chronic cigarette smoke (CS) exposure of air-liquid interface (ALI) cultures of primary bronchial epithelial cells (PBECs) changes cellular composition. a) ALI-PBECs were exposed during differentiation for 13-19 consecutive days to whole CS. Cells were lysed at several time points and RNA was isolated followed by complementary DNA synthesis, to assess gene expression of basal cell markers cytokeratin-5 (KRT5) and TP63, of early progenitor cell marker cytokeratin-8 (KRT8) and of specialised cell markers FOXJ1 (ciliated cells), SCGB1A1 (club cells) and MUC5B (goblet cells). Data are shown as target gene expression normalised for the geometric mean expression of the reference genes ATP synthase, hydrogen-transporting, mitochondrial F1 complex, $\beta$-polypeptide (ATP5B), $\beta 2$-microglobulin (B2M) and ribosomal protein L13a (RPL13A). Days 0,7 and $13 n=8$ donors; day $19 n=4$ donors. Significance was determined using a two-way ANOVA and Bonferroni post hoc test. **: $p<0.01$,

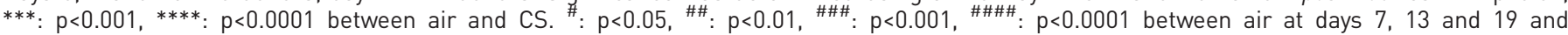
unexposed cultures at time 0. b) ALI-PBECs were differentiated for 2-3 weeks and exposed daily to CS. Subsequently, the cells were fixed in $1 \%$ paraformaldehyde and stained using primary antibodies against basal cells (p63) (red), in combination with primary antibodies against cytokeratin-8 (CK-8), acetylated $\alpha$-tubulin (ciliated cells), CC16 (club cells) and MUC5AC (goblet cells) (green); 4',6-diamidino-2-phenylindole (DAPI) was used to stain the nuclei (blue). Z-stacks and images of the apical and basal side of stained cells were obtained using confocal imaging. Images shown are representative for results obtained with cells from four different donors (CK-8, $\mathrm{n}=3$ different donors). Scale bars $=50 \mu \mathrm{m}$. 
a)
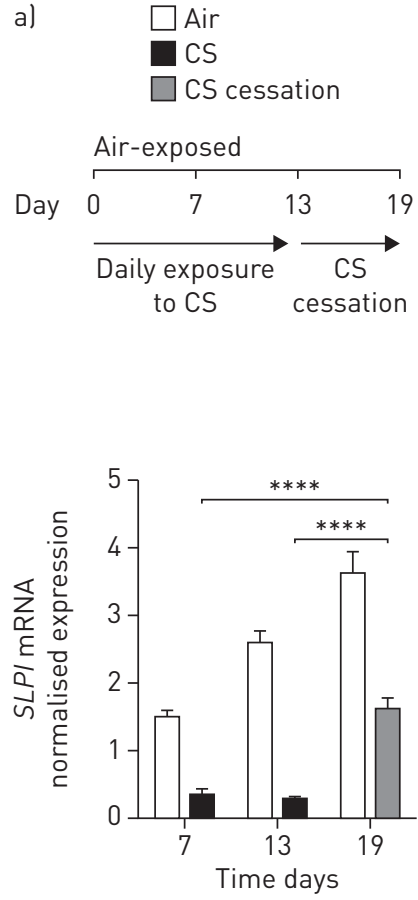

b)
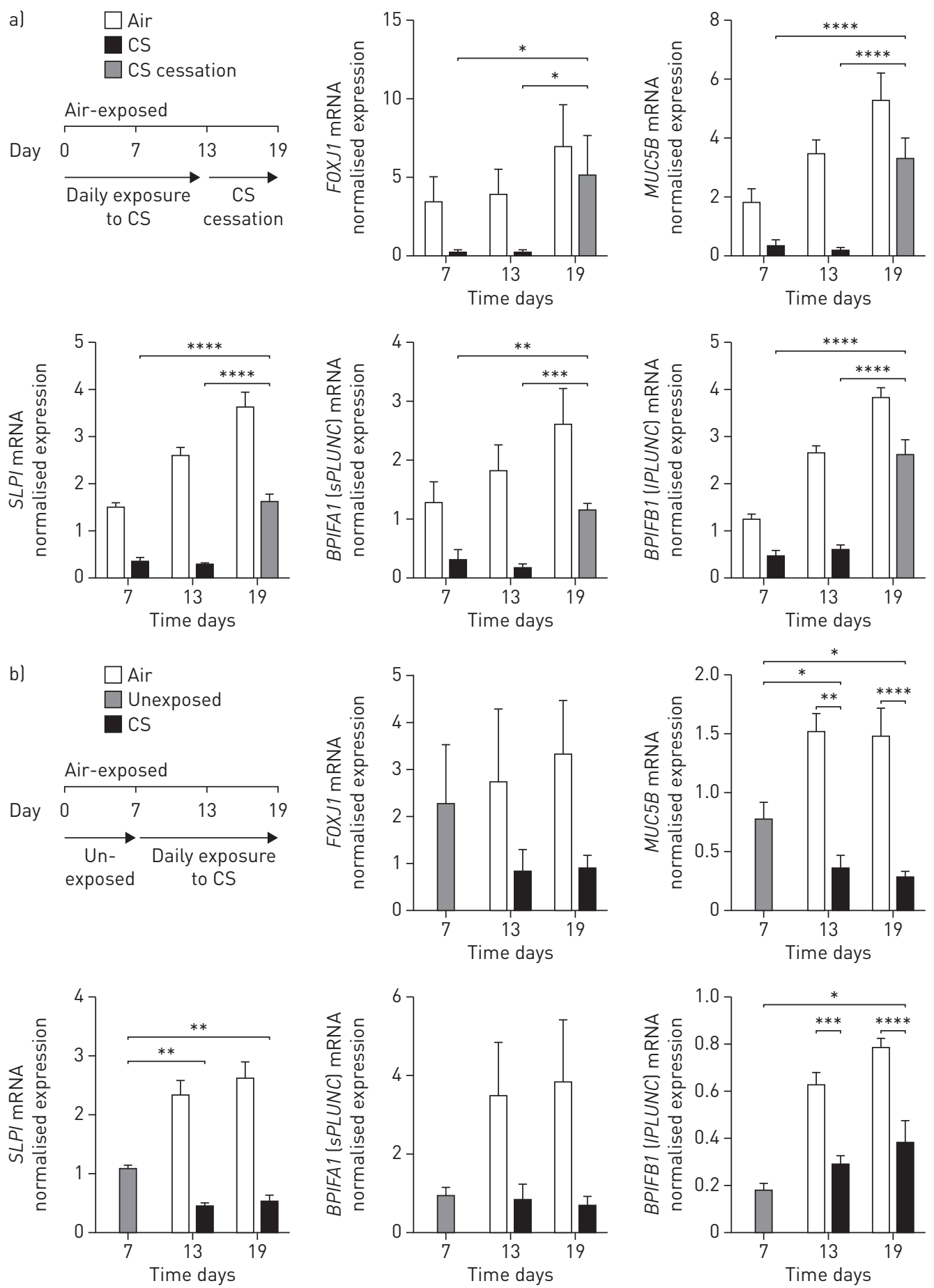
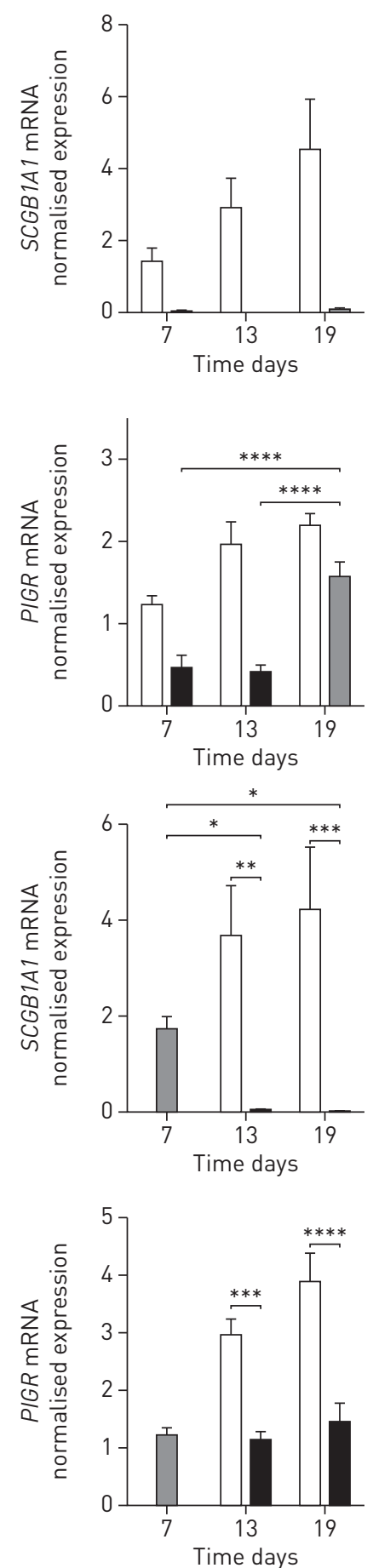

FIGURE 6 Cigarette smoke (CS)-induced impairment of host defence proteins and differentiation markers are partly persistent upon CS cessation. a) Air-liquid interface (ALI) cultures of primary bronchial epithelial cells (PBECs) were exposed during differentiation for 13 consecutive days to whole CS after which cultures were continued for another 6 days without CS exposure. Cells were lysed at several points during this course of time and RNA was isolated followed by complementary DNA synthesis, to assess gene expression of the cell specific markers: FOXJ1 (ciliated cells), MUC5B (goblet cells) and SCGB1A1 (club cells) and of respiratory defence proteins: SLPI (secretory leukocyte protease inhibitor), BPIFA1 (short palate, lung and nasal epithelium clone protein (sPLUNC)), BPIFB1 (long palate, lung and nasal epithelium clone protein (IPLUNC)) and $P I G R$ (polymeric immunoglobulin receptor). Data are presented as target gene expression normalised for the geometric mean expression of the reference genes ATP synthase, hydrogen-transporting, mitochondrial F1 complex, $\beta$-polypeptide (ATP5B), $\beta 2$-microglobulin (B2M) and ribosomal protein L13a (RPL13A). $\mathrm{n}=8$ different donors. Statistical differences were evaluated only for the difference between cessation and previous CS expression using a two-way ANOVA and Bonferroni post hoc test. b) ALI-PBECs were air-exposed at day 0 and cultured for 7 days under standard conditions. At day 7 cultures were exposed to CS for 12 consecutive days, after which the cells were lysed and analysed similarly to (a). Data are shown as target gene expression normalised for the geometric mean expression of the reference genes ATP5B, B2M and RPL13A. $\mathrm{n}=6$ different donors. Statistical differences were evaluated using a two-way ANOVA and Bonferroni post hoc test. *: $p<0.05,{ }^{* *}: p<0.01,{ }^{* * *}$ : $p<0.001$, $* * * *$ : $p<0.0001$. 

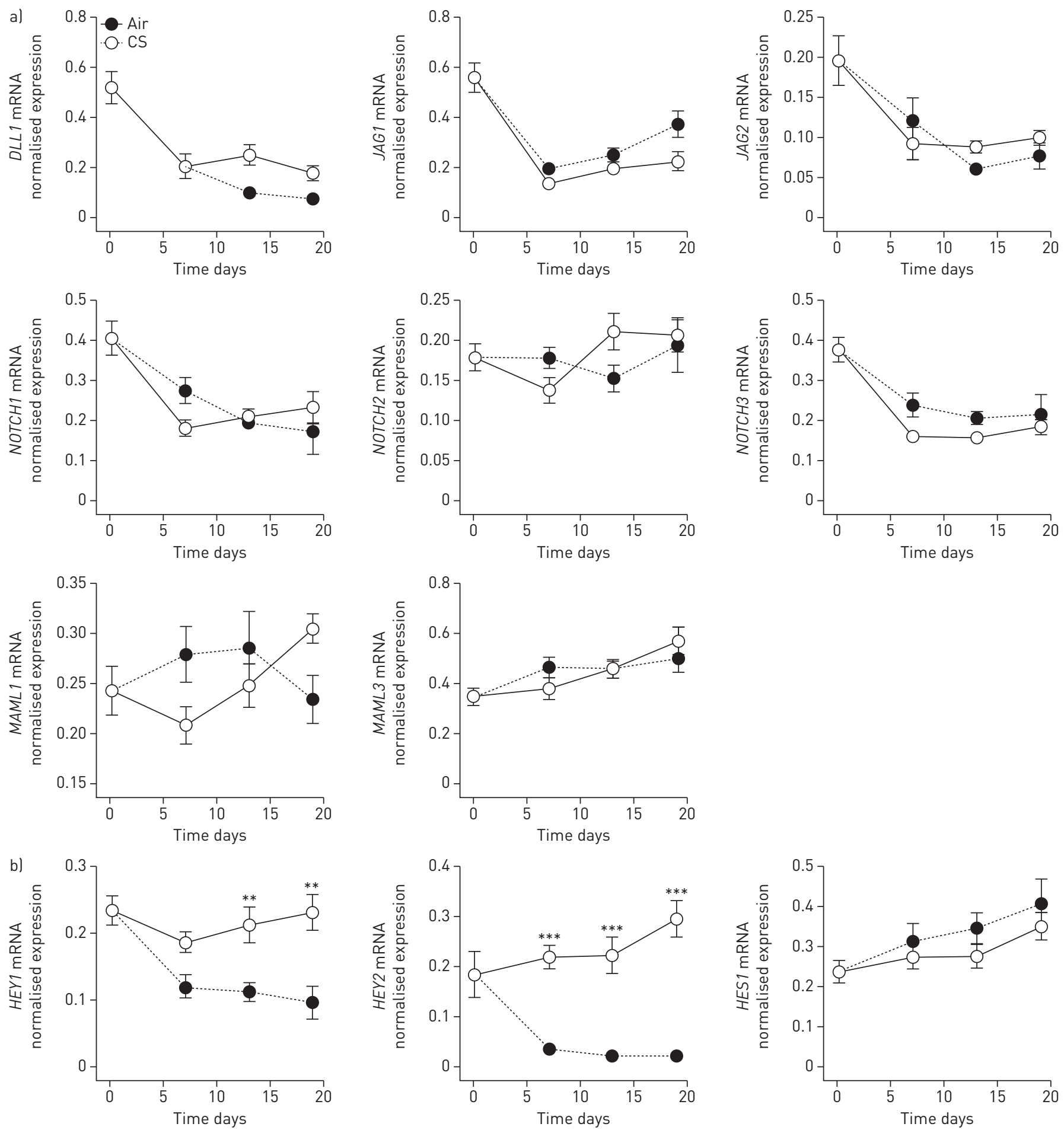

FIGURE 7 Chronic cigarette smoke (CS) exposure of air-liquid interface (ALI) cultures of primary bronchial epithelial cells (PBECs) results in selective impairment of Notch signalling. a) After 2 weeks of differentiation and daily cigarette smoke exposure, ALI-PBECs were lysed, RNA was isolated and complementary DNA synthesised. Subsequent quantitative (q)PCR analysis was performed on notch signalling ligands DLL1, JAG1 and JAG2, on Notch receptors 1-3 and on the transcriptional co-activators MAML1 and MAML3; data are shown as target gene expression normalised for the geometric mean expression of ATP synthase, hydrogen-transporting, mitochondrial F1 complex, $\beta$-polypeptide (ATP5B), $\beta 2$-microglobulin (B2M) and ribosomal protein L13a (RPL13A). $\mathrm{n}=8$ different donors. b) Subsequent qPCR analysis was performed on the Notch signalling target genes HEY1, HEY2 and HES1. Data are presented as target gene expression normalised for the geometric mean expression of the reference genes ATP5B, B2M and RPL13A. $\mathrm{n}=8$ different donors. Statistical significance was tested using a two-way ANOVA and Bonferroni post hoc test. ${ }^{* *}: p<0.01,{ }^{* * *}: p<0.001$ between air and CS. 
signalling (figure 8a). After 15 days of PBEC differentiation in the presence of DAPT, we measured expression of HDPs. DEFB1 (hBD-1) and LCN2 were not affected by DAPT, while gene expression of SLPI, BPIFA1 (sPLUNC), BPIFB1 (IPLUNC) and PIGR were strongly reduced by DAPT incubation (figure $8 \mathrm{~b})$. Furthermore, DAPT-exposed cultures showed a skewing of cell differentiation away from a secretory phenotype towards an increase in ciliated cells (figure 8c) that was also confirmed by confocal imaging (figure 8d).

\section{Discussion}

Here we demonstrate that cigarette smoke negatively affects expression of the respiratory HDPs: pIgR, SLPI, IPLUNC and sPLUNC. Their expression was significantly reduced in epithelial cells exposed daily to cigarette smoke during differentiation as a result of an impaired end-stage differentiation of specialised luminal cells. As a consequence, remodelling of the airway epithelium by cigarette smoke has a significant impact on respiratory host defence, underscored by the severely diminished IgA transport across the cigarette smoke-exposed epithelium and impaired antibacterial defences against $M$. catarrhalis and K. pneumoniae. Our data suggest that increasing expression of specific respiratory HDPs (or preventing their decrease) could be of therapeutic interest to improve host defences in the lungs of COPD patients. Furthermore, this (selective) loss may also contribute to changes in lung microbiome composition, which is increasingly recognised as an important contributor to chronic inflammatory lung diseases $[15,16]$.

The cellular composition of the ALI-PBEC cultures changed drastically upon chronic cigarette smoke-exposure. While presence of intermediate $\mathrm{CK}-8^{+}$cells (also called early, intermediate or committed progenitor epithelial cells $[13,17])$ was not affected by chronic cigarette smoke exposure, the specialised luminal cell markers were reduced in chronic cigarette smoke-exposed cultures. These results indicate that specifically end-stage differentiation seems impaired by cigarette smoke exposure. The effects of chronic cigarette smoke exposure were also observed when the cells were first allowed to differentiate for 1 week in absence of cigarette smoke. Furthermore, upon cessation of cigarette smoke exposure, gene expression of most luminal cell markers showed a strong recovery. In contrast, SCGB1A1 mRNA expression remained absent after almost 1 week of recovery, suggesting an exceptional detrimental effect of cigarette smoke on club cell differentiation or the regulation of SCGB1A1 gene expression. This is underscored by a study showing that expression of the club cell-protein CC16 (SCGB1A1) is reduced in COPD patients and in cigarette smoke-exposed mice. Loss of CC16 was correlated with increased severity of the disease and cigarette smoke-induced pulmonary inflammation was lower in mice overexpressing CC16 [18].

Cytotoxicity is unlikely to have a major contribution to the cigarette smoke-induced effects on the ALI-PBEC cultures since we detected no difference in transepithelial electrical resistance (TEER) as a measure of barrier function during the course of differentiation between cigarette smoke- and air-exposed controls. We previously observed transient decreases in TEER after acute single cigarette smoke exposures, normalising after $24 \mathrm{~h}$ [11]. In our chronic cigarette smoke set-up we measured TEER 18-20 h after the previous cigarette smoke exposure, which may explain why we did not observe significant differences in TEER between air- and cigarette smoke-exposed cultures. Previous studies have shown decreases in TEER by cigarette smoke, but often use cigarette smoke extract (CSE) and not whole cigarette smoke [19, 20]. CSE has a different composition and concentration to the whole cigarette smoke used in our study. In addition, in some studies CSE was added to the basal medium, resulting in stimulation from the basal side of the transwells [19]. This may also contribute to differences found in effects on TEER. Lactate dehydrogenase release was not increased in our cigarette smoke-exposed cultures, while cellular size appeared larger in the cigarette smoke-exposed cultures for some donors. Finally, the cigarette smoke-exposed cultures produced higher amounts of interleukin- 8 and displayed increased mRNA expression of the inducible antimicrobial peptides RNase7 and LL-37 (but not hBD-2). These results are described in the online supplementary material and in online supplementary figure S4.

Unexpectedly, we did not observe goblet cell hyperplasia in cultures that were exposed to cigarette smoke, shown previously in smokers [21], guinea pigs [22], rats [23] and in cell lines [23,24] or PBECs exposed to CSE [19]. However, BREKMAN et al. [25] observed a decline in goblet cells markers in PBECs continuously exposed to CSE. Data are therefore conflicting and dependent on the type of cell culture used. Obviously, in our primary differentiated cultures, whole cigarette smoke exposure alone is insufficient to induce goblet cell hyperplasia within 19 days. We strongly consider that the findings in patients are probably explained by a secondary effect of the cigarette smoke-induced inflammation (which is obviously incompletely represented in our in vitro model). For example, neutrophil recruitment as a consequence of the cigarette smoke-induced inflammation and subsequent release of proteases and other molecules may help to explain goblet cell formation in patients. In addition, the presence of other cell types besides neutrophils, such as macrophages, seem important for goblet cell hyperplasia. This has been suggested in the literature [26-29]. Furthermore, several studies suggest that various other factors that are 
a)
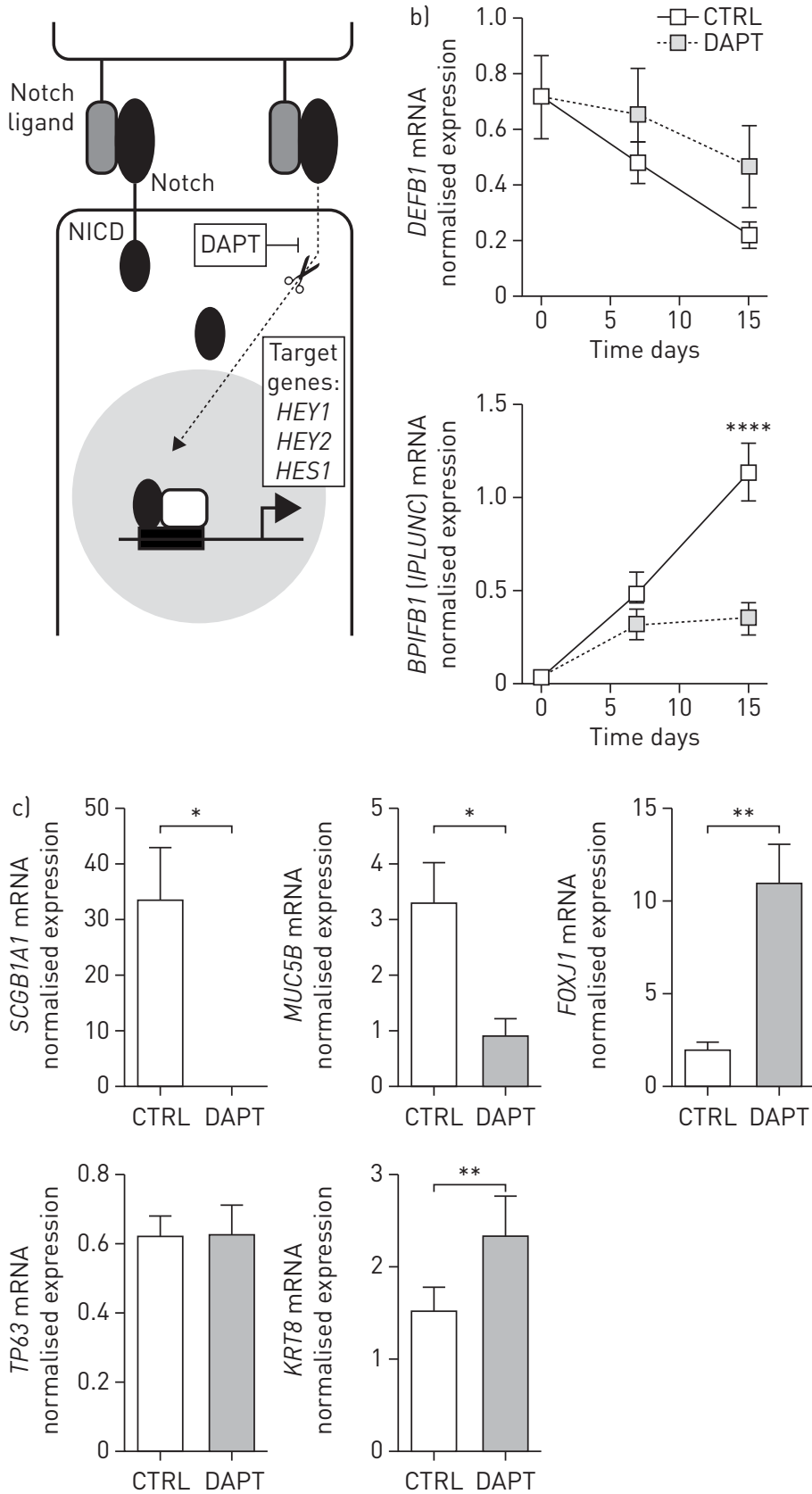
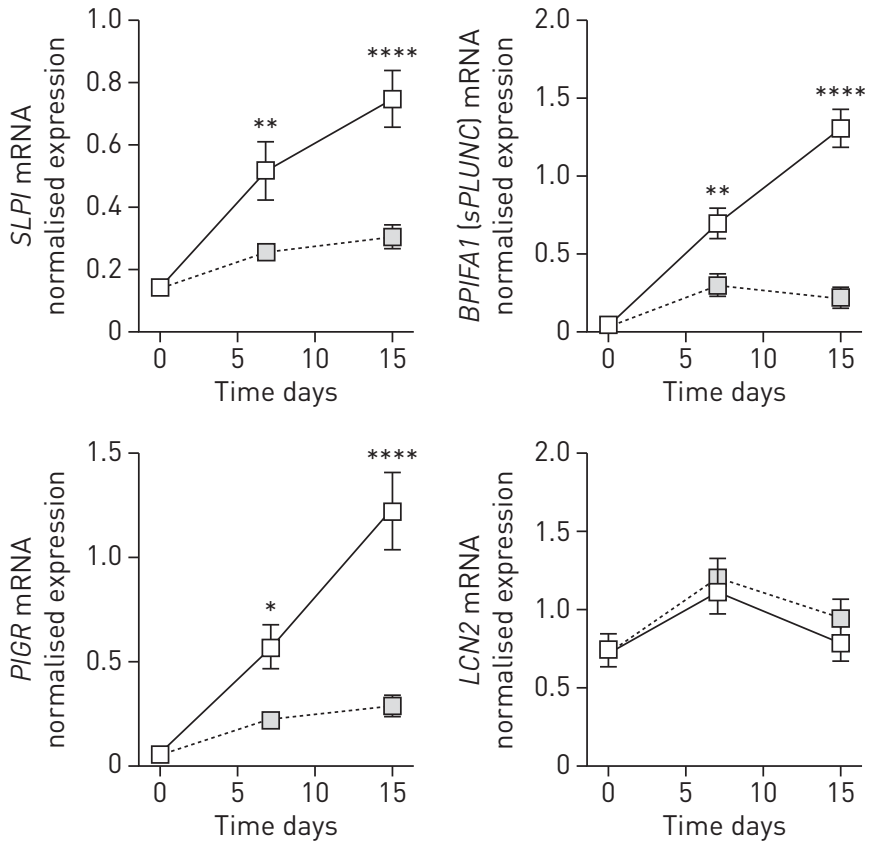

MUC5AC

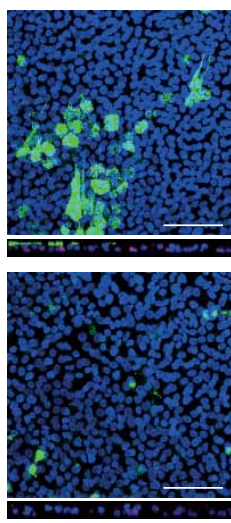

Acetylated

$\alpha$-tubulin

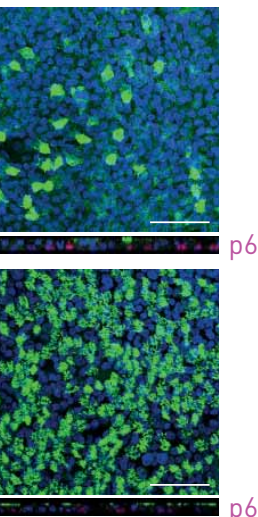

FIGURE 8 DAPT inhibits host defence protein expression in air-liquid interface (ALI) cultures of primary bronchial epithelial cells (PBECs). a) Mechanism of action of the Notch inhibitor DAPT, a $\gamma$-secretase inhibitor that prevents proteolytic cleavage of the Notch intracellular domain (NCID). b) PBECs were seeded on coated transwells and cultured in submerged conditions until confluent. At day 0 , cells were differentiated for an additional 15 days in the presence of $5 \mu \mathrm{M}$ of the Notch signal transduction inhibitor DAPT in the basal medium or solvent as control. At days 0 , 7, and 15 cells were lysed, RNA was isolated and complementary DNA synthesised. Subsequent quantitative (q)PCR analysis was performed to assess expression of respiratory defence proteins and epithelial cell-specific genes such as DEFB1 (human $\beta$ defensin-1), SLPI (secretory leukocyte protease inhibitor), BPIFA1 (short palate, lung and nasal epithelium clone protein), BPIFB1 (long palate, lung and nasal epithelium clone protein), polymeric immunoglobulin receptor (PIGR) and LCN2 (lipocalin 2). Data are shown as target gene expression normalised for the geometric mean expression of the reference genes ATP synthase, hydrogen-transporting, mitochondrial F1 complex, $\beta$-polypeptide (ATP5B), $\beta 2$-microglobulin (B2M) and ribosomal protein L13a (RPL13A). $\mathrm{n}=7$ different donors. Statistical significance was tested using a two-way ANOVA and Bonferroni post hoc test. ${ }^{*}: p<0.05,{ }^{* *}: p<0.01,{ }^{* * *}: p<0.0001$ between control (CTRL) and DAPT. c) qPCR analysis was performed to assess mRNA expression of the epithelial cell markers SCGB1A1 (club cells), MUC5B (goblet cells), FOXJ1 (ciliated cells), TP63 (basal cells) and cytokeratin-8 (KRT8) (intermediate cells) after 15 days of differentiation with DAPT or solvent control. $\mathrm{n}=6$ different donors. Statistical significance was tested using a paired t-test; *: $p<0.05,{ }^{* *}$ : $p<0.01$ between CTRL and DAPT. d) ALI-PBECs were differentiated for 15 days with DAPT or solvent control. Subsequently, the cells were fixed in 1\% paraformaldehyde and stained using primary antibodies against CC16 (club cells), MUC5AC (goblet cells) and acetylated $\alpha$-tubulin (ciliated cells) (green); 4',6-diamidino-2-phenylindole (DAPI) was used to stain the nuclei (blue). Images shown are representative for results obtained with cells from three different donors. Scale bars $=50 \mu \mathrm{m}$. 
involved in COPD pathogenesis, including bacterial and viral infections, might have an important role in promoting goblet cell hyperplasia [30, 31].

Whereas previous studies have shown that cigarette smoke reduces the presence of ciliated cells [19, 25, 32], so far cigarette smoke-induced airway epithelial remodelling has not yet been linked to changes in levels of the highly expressed respiratory HDPs, despite the fact that changes in expression in these proteins have been reported in smokers or patients with COPD. AARBIOU et al. [33] showed that expression of SLPI was significantly reduced in damaged bronchial epithelium of COPD patients compared to non-COPD individuals, and GoHY et al. [10] showed reduced levels of pIgR in the lungs of patients with COPD, compared to healthy controls; however, this was not seen in smokers with normal lung function. Finally, reduced levels of PLUNC were detected in bronchial brushes performed in current smokers compared to never-smokers [34].

We observed impaired antibacterial activity of the cigarette smoke-exposed airway epithelial cultures against $M$. catarrhalis and K. pneumoniae. In addition, we evaluated direct antibacterial activity of the chronic cigarette smoke-exposed cultures using a grid assay with live/dead staining of bacteria $[35,36]$ and via conventional plating methods against Pseudomonas aeruginosa and non-typeable Haemophilus influenzae, but could not detect any differences (data not shown). These data suggest that the observed cigarette smoke-induced impairment of antimicrobial activity may be pathogen-specific, since it is not observed with all pathogens studied. In addition to impaired antibacterial host defence activities, the loss of HDP expression by the airway epithelium can have further negative effects for the host. For example, loss of SLPI expression (highly expressed in normal lung tissue) can promote inflammation in the lungs of patients with COPD. SLPI acts as an inhibitor of detrimental proteases such as neutrophil elastase, acts as an inhibitor of nuclear factor- $\mathrm{\kappa B}$ activation and modulates macrophage functions [37-39]. sPLUNC is involved in the regulation of the epithelial sodium channel, thereby regulating airway surface liquid (ASL), and reduced levels could result in lowered ASL volume and impaired mucociliary clearance [40].

Since Notch signalling was previously reported to be impaired in COPD [14], we first analysed the effect of chronic cigarette smoke exposure on components of the Notch signalling pathway and Notch target genes, and found that cigarette smoke decreased the expression of selected Notch target genes. When we next used the Notch signalling inhibitor DAPT to inhibit airway epithelial cell differentiation, we found similar effects compared to cigarette smoke exposure on expression of the selected set of respiratory HDPs. To our knowledge, this is the first study linking Notch signalling to expression of a range of HDPs that are increased upon differentiation. Whereas chronic cigarette smoke exposure resulted in a reduction of all luminal cell markers, DAPT-exposed cultures showed higher levels of ciliated cells when compared to control-treated cells, in line with previous studies [41, 42]. These results suggest that expression of the luminal cell-restricted HDPs is confined to mature secretory epithelial cells, rather than the ciliated epithelium. Further studies using single-cell RNA sequencing, may reveal whether the expression is restricted to a certain secretory cell phenotype. The partial similarity between the DAPT-incubated cultures and the cigarette smoke-exposed cultures suggests involvement of altered Notch signalling in the cigarette smoke-induced effects. However, alterations in other signalling pathways might also be involved in the observed effects of cigarette smoke, such as those involving epithelial growth factor receptor [32], transforming growth factor- $\beta$ [25], Wnt [43] and bone morphogenetic protein [44]. It is likely that a more systems/-omics approach has the potential to elucidate in detail all effects of chronic cigarette smoke exposure on Notch and other signalling pathways [45].

In summary, these findings shed new light on the role of dysregulated host defence in smokers and patients with COPD by highlighting the importance of airway epithelial cell differentiation in the expression of respiratory HDPs. Further investigations into how suppression of epithelial cell differentiation by cigarette smoke contributes to microbial colonisation and infections of the airways are warranted in order to develop new therapeutics that restore airway epithelial host defence in patients with COPD.

Author contributions: Conception and design: G.D. Amatngalim, J.A. Schrumpf, F. Dishchekenian, P.S. Hiemstra, A.M. van der Does; analysis and interpretation: G.D. Amatngalim, J.A. Schrumpf, F. Dishchekenian, T.C.J. Mertens, D.K. Ninaber, A.C. van der Linden, C. Pilette, P.S. Hiemstra, A.M. van der Does; and drafting the manuscript for important intellectual content: G.D. Amatngalim, J.A. Schrumpf, C. Pilette, C. Taube, P.S. Hiemstra, A.M. van der Does.

Conflict of interest: P. Hiemstra reports grants (research fellowship) from the European Union (Marie Curie Intra-European Fellowship), and grants (unrestricted research grant) from Lung Foundation Netherlands, Galapagos N.V. and Boehringer Ingelheim, outside the submitted work.

Support statement: This work was supported by a Marie Curie Intra-European Fellowship (\#622815), and grants from the Lung Foundation of the Netherlands (\#5.1.13.033) and Galapagos N.V. Funding information for this article has been deposited with the Crossref Funder Registry. 


\section{References}

1 Sethi S. Infection as a comorbidity of COPD. Eur Respir J 2010; 35: 1209-1215.

2 Puchelle E, Zahm JM, Tournier JM, et al. Airway epithelial repair, regeneration, and remodeling after injury in chronic obstructive pulmonary disease. Proc Am Thorac Soc 2006; 3: 726-733.

3 Bals R, Hiemstra PS. Innate immunity in the lung: how epithelial cells fight against respiratory pathogens. Eur Respir J 2004; 23: 327-333.

4 Hiemstra PS, Amatngalim GD, van der Does AM, et al. Antimicrobial peptides and innate lung defenses: role in infectious and noninfectious lung diseases and therapeutic applications. Chest 2016; 149: 545-551.

5 Rojas R, Apodaca G. Immunoglobulin transport across polarized epithelial cells. Nat Rev Mol Cell Biol 2002; 3: 944-955.

6 McCray PB Jr, Bentley L. Human airway epithelia express a beta-defensin. Am J Respir Cell Mol Biol 1997; 16: 343-349.

7 Franken C, Meijer CJ, Dijkman JH. Tissue distribution of antileukoprotease and lysozyme in humans. J Histochem Cytochem 1989; 37: 493-498.

8 Bingle CD, Craven CJ. PLUNC: a novel family of candidate host defence proteins expressed in the upper airways and nasopharynx. Human Mol Genet 2002; 11: 937-943.

9 Rock JR, Randell SH, Hogan BL. Airway basal stem cells: a perspective on their roles in epithelial homeostasis and remodeling. Dis Model Mech 2010; 3: 545-556.

10 Gohy ST, Detry BR, Lecocq M, et al. Polymeric immunoglobulin receptor down-regulation in chronic obstructive pulmonary disease. Persistence in the cultured epithelium and role of transforming growth factor- $\beta$. Am J Respir Crit Care Med 2014; 190: 509-521.

11 Amatngalim GD, van Wijck Y, de Mooij-Eijk Y, et al. Basal cells contribute to innate immunity of the airway epithelium through production of the antimicrobial protein RNase 7. J Immunol 2015; 194: 3340-3350.

12 Wang $\mathrm{H}, \mathrm{Gu}$ X, Weng Y, et al. Quantitative analysis of pathogens in the lower respiratory tract of patients with chronic obstructive pulmonary disease. BMC Pulm Med 2015; 15: 94.

13 Rock JR, Gao X, Xue Y, et al. Notch-dependent differentiation of adult airway basal stem cells. Cell Stem Cell 2011; 8: 639-648.

14 Tilley AE, Harvey BG, Heguy A, et al. Down-regulation of the notch pathway in human airway epithelium in association with smoking and chronic obstructive pulmonary disease. Am J Respir Crit Care Med 2009; 179: $457-466$

15 Sze MA, Dimitriu PA, Suzuki M, et al. Host response to the lung microbiome in chronic obstructive pulmonary disease. Am J Respir Crit Care Med 2015; 192: 438-445.

16 Dickson RP, Erb-Downward JR, Martinez FJ, et al. The microbiome and the respiratory tract. Annu Rev Physiol 2016; 78: 481-504

17 Pan JH, Adair-Kirk TL, Patel AC, et al. Myb permits multilineage airway epithelial cell differentiation. Stem Cells 2014; 32: 3245-3256.

18 Laucho-Contreras ME, Polverino F, Gupta K, et al. Protective role for club cell secretory protein-16 (CC16) in the development of COPD. Eur Respir J 2015; 45: 1544-1556.

19 Schamberger AC, Staab-Weijnitz CA, Mise-Racek N, et al. Cigarette smoke alters primary human bronchial epithelial cell differentiation at the air-liquid interface. Sci Rep 2015; 5: 8163.

20 Xiao C, Puddicombe SM, Field S, et al. Defective epithelial barrier function in asthma. J Allergy Clin Immunol 2011; 128: 549-556.

21 Saetta M, Turato G, Baraldo S, et al. Goblet cell hyperplasia and epithelial inflammation in peripheral airways of smokers with both symptoms of chronic bronchitis and chronic airflow limitation. Am J Respir Crit Care Med 2000; 161: 1016-1021.

22 Komori $\mathrm{M}$, Inoue $\mathrm{H}$, Matsumoto $\mathrm{K}$, et al. PAF mediates cigarette smoke-induced goblet cell metaplasia in guinea pig airways. Am J Physiol Lung Cell Mol Physiol 2001; 280: L436-L441.

23 Takeyama K, Jung B, Shim JJ, et al. Activation of epidermal growth factor receptors is responsible for mucin synthesis induced by cigarette smoke. Am J Physiol Lung Cell Mol Physiol 2001; 280: L165-L172.

24 Shao MX, Nakanaga T, Nadel JA. Cigarette smoke induces MUC5AC mucin overproduction via tumor necrosis factor- $\alpha$-converting enzyme in human airway epithelial (NCI-H292) cells. Am J Physiol Lung Cell Mol Physiol 2004; 287: L420-L427.

25 Brekman A, Walters MS, Tilley AE, et al. FOXJ1 prevents cilia growth inhibition by cigarette smoke in human airway epithelium in vitro. Am J Respir Cell Mol Biol 2014; 51: 688-700.

26 Snider GL, Lucey EC, Christensen TG, et al. Emphysema and bronchial secretory cell metaplasia induced in hamsters by human neutrophil products. Am Rev Respir Dis 1984; 129: 155-160.

27 Lundgren JD, Kaliner M, Logun C, et al. Dexamethasone reduces rat tracheal goblet cell hyperplasia produced by human neutrophil products. Exp Lung Res 1988; 14: 853-863.

28 Arai N, Kondo M, Izumo T, et al. Inhibition of neutrophil elastase-induced goblet cell metaplasia by tiotropium in mice. Eur Respir J 2010; 35: 1164-1171.

29 Silva MA, Bercik P. Macrophages are related to goblet cell hyperplasia and induce MUC5B but not MUC5AC in human bronchus epithelial cells. Lab Invest 2012; 92: 937-948.

30 Londhe V, McNamara N, Lemjabbar H, et al. Viral dsRNA activates mucin transcription in airway epithelial cells. FEBS Lett 2003; 553: 33-38.

31 Chu HW, Jeyaseelan S, Rino JG, et al. TLR2 signaling is critical for Mycoplasma pneumoniae-induced airway mucin expression. J Immunol 2005: 174: 5713-5719.

32 Valencia-Gattas M, Conner GE, Fregien NL. Gefitinib, an EGFR tyrosine kinase inhibitor, prevents smoke-mediated ciliated airway epithelial cell loss and promotes their recovery. PLoS One 2016; 11: e0160216.

33 Aarbiou J, van Schadewijk A, Stolk J, et al. Human neutrophil defensins and secretory leukocyte proteinase inhibitor in squamous metaplastic epithelium of bronchial airways. Inflamm Res 2004; 53: 230-238.

34 Steiling K, Kadar AY, Bergerat A, et al. Comparison of proteomic and transcriptomic profiles in the bronchial airway epithelium of current and never smokers. PLoS One 2009; 4: e5043.

35 Schrumpf JA, Amatngalim GD, Veldkamp JB, et al. Proinflammatory cytokines impair vitamin D-induced host defense in cultured airway epithelial cells. Am J Respir Cell Mol Biol 2017; 56: 749-761. 
36 Amatngalim GD, Schrumpf JA, Henic A, et al. Antibacterial defense of human airway epithelial cells from chronic obstructive pulmonary disease patients induced by acute exposure to nontypeable Haemophilus influenzae: modulation by cigarette smoke. J Innate Immun 2017; 9: 359-374.

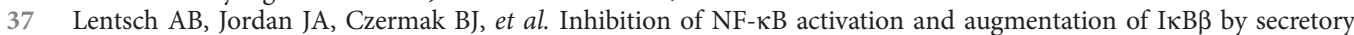
leukocyte protease inhibitor during lung inflammation. Am J Pathol 1999; 154: 239-247.

38 Sallenave JM, Si Tahar M, Cox G, et al. Secretory leukocyte proteinase inhibitor is a major leukocyte elastase inhibitor in human neutrophils. J Leukoc Biol 1997; 61: 695-702.

39 Ding A, Thieblemont N, Zhu J, et al. Secretory leukocyte protease inhibitor interferes with uptake of lipopolysaccharide by macrophages. Infect Immun 1999; 67: 4485-4489.

40 Garcia-Caballero A, Rasmussen JE, Gaillard E, et al. SPLUNC1 regulates airway surface liquid volume by protecting ENaC from proteolytic cleavage. Proc Natl Acad Sci USA 2009; 106: 11412-11417.

41 Gerovac BJ, Valencia M, Baumlin N, et al. Submersion and hypoxia inhibit ciliated cell differentiation in a notch-dependent manner. Am J Respir Cell Mol Biol 2014; 51: 516-525.

42 Konishi S, Gotoh S, Tateishi K, et al. Directed induction of functional multi-ciliated cells in proximal airway epithelial spheroids from human pluripotent stem cells. Stem Cell Reports 2016; 6: 18-25.

43 Lemjabbar-Alaoui $\mathrm{H}$, Dasari V, Sidhu SS, et al. Wnt and Hedgehog are critical mediators of cigarette smoke-induced lung cancer. PLoS One 2006; 1: e93.

44 Heijink IH, de Bruin HG, van den Berge M, et al. Role of aberrant WNT signalling in the airway epithelial response to cigarette smoke in chronic obstructive pulmonary disease. Thorax 2013; 68: 709-716.

45 Wöltje K, Jabs M, Fischer A. Serum induces transcription of Hey1 and Hey2 genes by Alk1 but not Notch signaling in endothelial cells. PLoS One 2015; 10: e0120547. 